\title{
Autonomic Dysfunction Following Neurotoxic Snake Envenomation: Does Site of Bite Predispose?
}

\author{
Anju Bala' ${ }^{1}$, Parul Bhardwaj', ${ }^{2}$ ipan Garg ${ }^{3}$, Shivbrat $^{4}$ \\ ${ }^{1}$ MD Pediatrics, Civil Hospital Nadaun (H.P.) \\ ${ }^{2}$ MD Internal Medicine, Civil Hospital Dehra (H.P.) \\ ${ }^{3}$ Senior Resident Anesthesia, DRPGMC Kangra at Tanda (H.P.) \\ ${ }^{4}$ Junior Resident ENT, DRPGMC Kangra at Tanda (H.P.) \\ Corresponding Author: Parul Bhardwaj
}

\begin{abstract}
South Asian subcontinent is among the highest burden areas in respect of snake bite. India is commonest place in this subcontinent with mortality following snake envenomation. The four most important venomous snakes in India are cobra (Naja naja) and common krait (Bungarus caeruleus), which are neurotoxic, and the saw-scaled viper (Echis carinatus) and Russell's viper (Daboia russelii), which are hemotoxic ${ }^{1}$. Various autonomic dysfunction following neuro-paralytic envenomation with krait and cobra has been reported. Timely recognition and appropriate management of snake envenomation along with autonomic dysfunction helps to reduce morbidity and mortality.
\end{abstract}

Keywords: Autonomic dysfunction, hypertension, antisnake venom, blood pressure

\section{INTRODUCTION}

With an estimate of mortality of 35,000-50,000 people per annum, snake envenomation is commonest medical emergency in India ${ }^{2}$. Neuroparalysis due to envenomation by common cobra (Naja naja) and common krait (Bungarus caeruleus) is a common life threatening medical emergency 11. The commonly encountered venomous snakes in India include common cobra (Naja naja), common krait (Bungarus caeruleus), Russell's viper (Daboia russelii) and saw scaled viper (Echis carinatus), against which a locally prepared equine polyvalent ASV antitoxin is available 3 . Neurological manifestations that follow envenoming by elapids (cobras and kraits) and less commonly Russell's viper include ptosis, external ophthalmalgia, paralysis of pharyngeal muscles, followed by respiratory and generalized muscle paralysis ${ }^{3}$. The acute clinical effects of snakebites vary from mild local reactions to severe, lifethreatening systemic responses depending on the species and size of the snake; the location of the bite; the amount of venom inoculated; and the age, weight, and wellbeing of the patient ${ }^{4}$. Due to higher venom volume to body surface ratio in children, they are more prone to severe envenomation and autonomic instability. Here we present a case of 5 years old male child who presented neurotoxic snake bite with autonomic dysfunction in the form of hypertension.

\section{CASE REPORT}

A 5 years old male child presented with history of abdominal pain, vomiting, pooling of secretions from mouth, drooping of eyelids, difficulty in breathing to hospital during morning hours. These all symptoms develop while child was sleeping. As per history provided by parents, child develops these symptoms soon after waking up. At the time of physical examination, child was drowsy with regular heart rate of 160 beats per min, shallow irregular respiratory efforts and blood pressure of 110/60 $\mathrm{mm} \mathrm{Hg}$ (> 99 percentile) in right arm supine position. His 
oxygen saturation was $50 \%$ on room air. Fang marks of snake bite were noted behind right ear. On further evaluation of symptoms, history and examination diagnosis of neurotoxic snake bite was made. Child was immediately intubated and was started on mechanical ventilation. There was no abnormal finding on abdominal and respiratory system. Investigations including complete blood count, serum electrolytes, renal function test, liver function test, coagulation profile, whole blood clotting time and urine routine and microscopic examination were normal. Child was administered with ten vials of polyvalent ASV. In view of no improvement in condition of child after 30 minutes again ten vials of ASV were administered with a total dose of 20 vials. Even after giving adequate sedation there was no improvement in hypertension. As there was no prior history of episodes of hypertension, we kept a possibility of autonomic dysfunction secondary to neurotoxic snake bite. Child was started on nitroglycerine infusion for hypertensive urgency. Patients remain on infusion for 12 hours and then infusion tapered off gradually. After 48 hours the neurological status of patient was improved and patient was extubated on $3^{\text {rd }}$ day of mechanical ventilation. To rule out secondary causes of hypertension different investigations done including chest radiograph, echocardiography, ultrasound abdomen, renal Doppler and urine catecholamine. All investigations came out to be unremarkable. After five days of hospitalization, patient was discharged. Blood pressure remains normal during follow-up.

\section{DISCUSSION}

Approximately 35,000 people die every year following snake envenomation in India, in which most common cause is delay in early diagnosis and medical treatment ${ }^{2}$. Presynaptic blockade by krait toxin and post synaptic blockade by cobra toxin are attributed in Neuroparalysis caused by these snakes ${ }^{1}$. Envenomation with these snakes may presents as drooping of eyelid, external ophthalmalgia, difficulty in swallowing, respiratory and generalized muscle weakness and associated with an overall mortality of $3.5 \%{ }^{5}$. Autonomic dysfunction in snakebite may present as abdominal pain, vomiting, mild-to-moderate hypertension or hypotension and cardiac arrhythmia. Cause of autonomic dysfunctions is not clear and still under study. However, Alpha-2 adrenergic blockade at presynaptic level which block release of norepinephrine can be attributed to the cause ${ }^{6}$. Hence, this process gives rise to sympathetic overactivity and decreased parasympathetic stimulation ${ }^{6-8}$. The $\alpha$ - and $\beta$-bungarotoxin of krait mainly inhibits the release of acetylcholine causing paralysis, but cases have been reported with the possibility of pulmonary edema along with ventricular fibrillation and also fulminant myocarditis 12,13. Hypertension without neurotoxic symptoms was observed in patients with Western Russell's viper envenomation ${ }^{10}$, and neurotoxic signs without hypertension were observed in an episode of the Eastern Russell's viper envenomation. It therefore appears that different toxins are responsible for cardiovascular and neurological symptoms ${ }^{9}$. In Our case definite diagnosis about type of snake cannot be made but as per presentation and bite mark it seems to be krait. In a study of common krait bites, 139 of 210 victims $(66 \%)$ exhibited autonomic dysfunction, which was more marked in those with severe envenomation ${ }^{6}$. Although snake was not seen by any family member but it is well known that kraits are active and agile at night, and during the rainy season, they frequently seek refuge in dry places, such as those inside a house or dwelling place ${ }^{14}$. Additionally, if humans are bitten by krait during their sleep, they are seldom aware of it, as their experience of the bite generally resembles that from an ant or a mosquito ${ }^{14}$.

\section{CONCLUSION}

Early diagnosis and management is important to reduce morbidity and mortality 
in snake envenomation. In children, lack of proper history makes it difficult for physician to ascertain the diagnosis. High dose of venom in comparison to body make them more prone to envenomation and autonomic dysfunction. Site of bite may also correlate with autonomic instability in our case, though not mentioned anywhere and need further research. For better outcome, limb immobilization, prompt ASV administration and ventilator support should be there along with vigilant monitoring for autonomic dysfunction.

\section{Declaration of patient consent:}

The authors certify that they have obtained all appropriate patient consent forms regarding images and other clinical information to be reported in the journal.

\section{Acknowledgement: None}

Conflict of Interest: There are no conflicts of interest.

\section{Source of Funding: None}

\section{REFERENCES}

1. Singh A, Balasubramanian V, Gupta N. Autonomic Dysfunction Manifesting as Severe Hypertension Following Cobra Envenomation: An Unusual Occurrence. J Emerg Med Case Rep 2018; 9: 51-4.

2. WHO/SEARO guidelines for the clinical management of snake bites in the Southeast Asian region. Southeast Asian J Trop Med Public Health. 1999;30(suppl 1):1-85.

3. Warrell DA. Snake bite, Lancet, 2010, vol. 375 (pg. 77-88)

4. Juckett G, Hancox JG. Venomous snakebites in the United States: management review and update. Am Fam Physician. 2002;65:1367-78.

5. Agarwal R, Aggarwal AN, Gupta D. Elapid snakebite as a cause of severe hypertension. J Emerg Med. 2006; 30: 319-20.
6. Kularatne S.A. Common krait (Bungarus caeruleus) bite in Anuradhapura, Sri Lanka: a prospective clinical study, 1996-98. Postgrad Med J. 2002;78:276-280.

7. Agarwal R., Aggarwal A.N., Gupta D. Elapid snakebite as a cause of severe hypertension. J Emerg Med. 2006;30:319320.

8. Laothong C., Sitprija V. Decreased parasympathetic activities in Malayan krait (Bungarus candidus) envenoming. Toxicon. 2001;39:1353-1357.

9. Malina T., Krecsak L., Warrell D.A. Neurotoxicity and hypertension following European adder (Vipera berus berus) bites in Hungary: case report and review. QJM. 2008;101:801-806.

10. Hung D.Z., Wu M.L., Deng J.F., Yang D.Y., Lin-Shiau S.Y. Multiple thrombotic occlusions of vessels after Russell's viper envenoming. Pharmacol Toxicol. 2002;91: 106-110

11. Kasturiratne A, Wickramsinghe AR, DeSilva N, Gunawardena NK, Pathmeswaran A, Premaratna R, et al. The global burden of snakebite: A literature analysis and modelling based on regional estimates of envenoming and deaths. PLoS Med 2008; 5: e218.

12. Pillai LV, Ambike D, Husainy S, Khaire A, Captain A, Kuch U. Severe neurotoxic envenoming and cardiac complications after the bite of a 'Sind Krait' (Bungarus cf. sindanus) in Maharashtra, India. Trop Med Health 2012;40:103-8.

13. Verma VK, Maurya V, Verma R. Indian common krait envenomation presenting as fulminant myocarditis and coma: A case report. Int J Res Med Sci 2017;2:1713-7.

14. Meenakshisundaram R, Senthilkumaran S, Thirumalaikolundusubramanaian P. Severe hypertension in elapid envenomation. J Cardiovasc Dis Res 2013;4(1): 65-67.

How to cite this article: Bala A, Bhardwaj P, Garg V et.al. Autonomic dysfunction following neurotoxic snake envenomation: does site of bite predispose? International Journal of Science \& Healthcare Research. 2021; 6(2): 4749. DOI: https://doi.org/10.52403/ijshr. 20210409 